\title{
Clinical Significance of Cancer Stem Cell Markers CD133 and CXCR4 in Osteosarcomas
}

\author{
Azam Mardani ${ }^{1}$, Elmira Gheytanchi², Seyed Hamzeh Mousavie ${ }^{3}$, Zahra Madjd \\ Jabari $^{2}$, Tina Shooshtarizadeh ${ }^{1 *}$
}

\begin{abstract}
Objective: Osteosarcomas (OS) is one the most common primary bone malignancy in humans with the lungs metastasis in most cases. Metastasis and recurrence of OS is attributed to cancer stem cells (CSCs). Our study aimed to evaluate the clinical significance of $C D 133$ and C-X-C chemokine receptor type $4(C X C R 4)$ as the frequently applied markers for CSCs in OS patients. Methods: In this cross-sectional, a total of 50 tissue samples from the patients with primary OS were immunohistochemically examined to detect the expression of CD133 and CXCR4. The associations of the relative expression and clinical significance of each marker were also evaluated. Results: High level expression of $C D 133$ was detected in $26 \%$ of OS patient tissues. Of the 12 patients who showed lung metastasis, 5 cases showed high expression of $C D 133$ with marginal trend correlation $(\mathrm{P}=0.06)$. No significant correlation was observed between CD133 expression and clinicopathological factors. Only 36\% of cases showed CXCR4 expression which was not significantly correlated with gender, age, tumor size, necrosis, stage and metastasis $(\mathrm{P}>0.05)$. Clinically, patients with concomitant $C D 133 / C X C R 4$ expression had significant association with lung metastasis $(\mathrm{P}=0.05)$. Conclusion: Our findings showed that concomitant expression of CSC markers CD133/CXCR4 might had a synergistic effect on the OS poor prognosis. These markers could be considered as potential therapeutic candidates of OS targeted therapy.
\end{abstract}

Keywords: Osteosarcoma- CD133- CXCR4- bone cancer- prognosis

Asian Pac J Cancer Prev, 21 (1), 67-73

\section{Introduction}

Bone cancers are a group of mesenchymal malignancies that include osteosarcoma (OS) and chondrosarcoma with clinical, histological, and molecular heterogeneity (Gibbs et al., 2005). OS, also called osteogenic sarcoma, is the most common malignant primary bone tumor in humans which is exhibited with the lungs micro metastasis as the main causes of OS deaths at diagnosis in approximately 95\% of the patients (Adhikari et al., 2010). The most common sites for OS are the distal femoral, proximal tibia and proximal humeral metaphysis (Lu et al., 2015c). The age-standardized incidence of osteosarcoma is estimated at approximately 5 per million annually which is typically occurs in children, adolescents and young adults (He et al., 2012; Ottaviani and Jaffe, 2009). The lung considered as the most common distant metastatic site for OS with a high-grade histopathology.

Potential for spontaneous pulmonary metastasis has been reported in the established osteosarcoma cell lines such as 9607-F5M2 (F5M2) with high tumorigenic ability. Despite multidisciplinary therapeutic strategies including intensive or neoadjuvant chemotherapy specially for high-grade osteosarcoma after aggressive surgical resection (mostly limb-sparing) and radiotherapy, the survival rate of osteosarcoma patients is still low (Adhikari et al., 2010). Therefore, in order to design appropriate therapeutic strategies and to overcome OS tumor progression, it is essential to understand the molecular mechanisms underlying chemo resistance and metastasis (Tobe et al., 2017).

It has been suggested that OS cancer stem cells (CSCs) may play roles in tumor recurrence, metastasis and chemo resistance (Yan et al., 2016). CSCs are a subpopulation of tumor cells with self-renewal and differentiation properties that can sustain tumor growth and recapitulate a heterogeneous tumor (Keymoosi et al., 2014; Madjd et al., 2013). Treatment failure and recurrence in several cancers including OS are attributed to the lack of ability in CSCs targeting (Adhikari et al., 2010). Therefore finding novel diagnostic and prognostic biomarkers to target these cells result in the successful treatment of OS (Yan et al., 2016). The functional methods of CSC detection in different types of sarcomas based on cell surface markers and also in stem-like gene expression levels have been studied in previous studies (Gibbs et al., 2005; Diestra et al., 2002; Murase et al., 2009; Suvà et al., 2009; Adhikari et al., 2010; Terry and Nielsen, 2010; Sana et al., 2011). 
Different stem-like genes and markers including ALDH, CD133, CD117/Stro1, CD271, Oct-4, NANOG, $C X C R 4$, nestin and $A B C G 2$ related with the CSC phenotype have been reported by previous studies (Brown et al., 2017; Honoki et al., 2010; Wang et al., 2011; Tirino et al., 2008; Tirino et al., 2011; Adhikari et al., 2010). Adhikari et al evaluated mesenchymal stem cell (MSC) markers such as CD117 and Stro-1in OS CSCs duo to its mesenchymal origin and found that theses markers could be easily initiated tumors in immunocompromised mice (Adhikari et al., 2010). They showed that all of CD117+ highly metastatic cells with drug-resistant properties were positive for $C X C R 4$ and $A B C G 2$ (Adhikari et al., 2010). One of CSC markers which involved in hematopoietic stem cell (HSC) mobilization (Gelmini et al., 2008) and homing and other cancers metastasis (Zhang et al., 2012; Hermann et al., 2007) is the chemokine SDF1/ CXCL12 and its receptor CXCR4 (Adhikari et al., 2010). The stromal cell-derived factor-1 (SDF-1/CXCR4) axis has been considered as a seven-transmembrane $\mathrm{G}$ protein-coupled receptor which led to tumor metastasis in several cancers (Kucia et al., 2005; Müller et al., 2001; Saur et al., 2005; Zeelenberg et al., 2003; Lu et al., 2015b). Therefore, CXCR4 expression has been linked to tumor cell invasiveness (Wang et al., 2015; Song et al., 2015; Perissinotto et al., 2005; Sun et al., 2010) which is also expressed by osteoblasts and by malignant cells in osteosarcoma (Jung et al., 2006).

Therefore, cumulative evidence proposes a critical role of CXCR4 as a CSC marker in the metastasis. It has been shown that $C X C R 4$ was expressed and enriched in $C D 133$ glioblastoma CSCs (Liu et al., 2006). CD133 (also known as prominin-1/ AC133), is a member of the Penta span transmembrane glycoproteins and a cell surface marker with a molecular weight of $120 \mathrm{kDa}$ (Madjd et al., 2016; Madjd et al., 2013). It is firstly expressed in hematopoietic stem cells (He et al., 2012) and is also considered as the most important surface marker for identification of CSCs in various solid tumors, such as hepatocarcinoma (Yin et al., 2007), colorectal cancer (Kazama et al., 2017), lung cancer (Roudi et al., 2015), transitional cell carcinomas (Sedaghat et al., 2017), synovial sarcoma and melanoma (He et al., 2012; Madjd et al., 2013). He et al showed that high expression of CD133 in OS tissues could predicts lung metastasis, poor prognosis and short survival time in OS patients which were correlated with higher expression of other well-known CSC markers including Oct-4, NANOG and CXCR4 in gene expression levels (He et al., 2012). Current study is focused on the immunohistochemically analysis of CXCR4 and CD133 expression in osteosarcoma as the most frequent types of adult and pediatric sarcomas. Up to now, this is the first report concerning combine analysis of these two putative CSC markers (CXCR4/CD133) in osteosarcomas. Moreover, we also evaluated the possible relationship of CXCR4 and CD133 expression in osteosarcoma samples.

\section{Materials and Methods}

\section{Patient's data collection and tumor characteristics}

In this cross-sectional, formalin- fixed-paraffin- embedded (FFPE) OS sections from 50 patients who were diagnosed with primary OS and who underwent tumor resection between 2011-2016 years were obtained from the Shafa-Yahyaian Hospital, a major university-based and referral center in Tehran, Iran.

All patients had written informed consent for participation in the present study. Furthermore, the ethical use of patients' tissue blocks and the chart reviews were approved by the Ethics Committee of the Iran University of Medical Sciences.

Postoperative adjuvant chemotherapy [four/six courses of Ifosfamide ( $2 \mathrm{~g} / \mathrm{m}^{2}$ for 5 days/course), methotrexate ( $8 \mathrm{~g} /$ $\mathrm{m}^{2}$ for 1 day/course) and Adriamycin $\left(50 \mathrm{mg} / \mathrm{m}^{2}\right.$ for 1 day/ course)] were only prescribed in patients with Enneking stage I, II, III or IV disease. Patient's medical records were reviewed to collect relevant clinical data, including gender, age, tumor location, tumor size, Enneking stage, necrosis, local recurrence and lung metastasis status. All hematoxylin and eosin stained tumor slides were evaluated for diagnosis by experienced pathologist.

\section{Immunohistochemistry (IHC) assay}

The level of CD133 and CXCR4 expression was examined in OS samples by IHC technique, as described previously (Foroozan et al., 2017; Sedaghat et al., 2017). Briefly, paraffin-embedded tissues (5- $\mu \mathrm{m}$ thickness) were mounted onto Super frost slides (Superfrost plus, Thermo Scientific, Germany), dewaxed at $60^{\circ} \mathrm{C}$ for 30 min, deparaffinized in xylene and rehydrated in different concentrations of ethanol. The sections then were treated by $3 \%$ hydrogen peroxide for $20 \mathrm{~min}$ to quench the endogenous peroxidase activity. Antigenic retrieval process was performed by submerging in Tris-EDTA ( $\mathrm{pH}$ 8.0) citrate buffer ( $\mathrm{pH} \mathrm{6.0)}$ as a target retrieval solution of CXCR4 and CD133, respectively to damask antigenic epitope. The slides were then allowed to cool at room temperature, followed by rinsing in Tris-buffered saline (TBS) and then in protein block serum-free (Dako; code X0909; Denmark; Ready-to-use) to decrease nonspecific binding and background staining for 10 min. For primary staining, all slides were incubated in monoclonal mouse anti-human CD133 antibody (1:100, overnight at $4^{\circ} \mathrm{C}$, Sina Biotech; SB-019762; Iran) and monoclonal mouse anti-human CXCR4/CD184 (ready to-use, $60 \mathrm{~min}$ at RT, Medaysis; code RM0407RTU7), respectively. For secondary staining, EnVisionTM/ HRP, Rabbit/Mouse (ENV) reagent (Dako; code K5007; Denmark; Ready to-use) and then visualized by Dako REALTM DAB + Chromogen (Dako; Denmark) according to the manufacturer's instructions. After washing, the sections were followed by counterstaining with Mayer's hematoxylin dye (Dako; Denmark) for $15 \mathrm{~min}$, dehydrated in different degrees of alcohol and cleared in xylene. Human tonsil and kidney tissues were used as positive controls of CXCR4 and CD133 antibodies, respectively. For negative control staining the primary antibodies were omitted.

Evaluation and scoring system of immunohistochemistry

Each stained sections were evaluated by two independent pathologist observers (T.S. and A.M.) without 
knowledge of the patient's outcome and other clinical characteristics and scored based on the intensity and extensity of staining, and histochemical score (H-score) by multiplying the staining intensity and extensity of positive tumor cells. For each marker, membranous and cytoplasmic immunostaining was considered to be positive. The scoring of CD133 staining (0-300) was classified as the staining extension (0-100) multiplied by the staining intensity (0-3). The extensity of CXCR4+ tumor cells (TC) was categorized into four levels: 1 (25 $\%$ CXCR4+ TC), 2 (26-50\% CXCR4+ TC), 3 (51-75 $\%$ CXCR4+ TC), and 4 (76-100\% CXCR4+ TC). The intensity of immunostaining was classified as no staining $(0 /-)$, weak or only visible at high magnification $40 \times$ $(1+)$, moderate or visible at low magnification $10 \times(++)$, and strong at low magnification $10 \times(+++)$. The whole sections were screened under light microscope at $\times 400$ magnification and were classified into Low or high expression levels of CXCR4 and CD133 based on the median of H-scores as a cut-off value (Keymoosi et al., 2014).

\section{Statistical analyses}

Each experiment was analyzed using the SPSS software version 20 (SPSS, Chicago, IL, USA). Values were stated as mean $\pm \mathrm{SD}$. Correlation of each marker with clinicopathological features of patients was evaluated using Pearson's chi-square or Fisher's exact test, where appropriate. The one-way ANOVA and Tukey post hoc analysis was applied to examine the association between CD113/CXCR4 phenotypes and clinicopathological variables. A value of $\mathrm{P}<0.05$ was considered statistically significant.

\section{Results}

Patient clinicopathological characteristics

The mean age of all OS cases was $19 \pm 8.926$ years ranging from 10-60 and 24 (48\%) patients were older than 19 years of age. Twenty-nine $(58 \%)$ of patients were male and $21(42 \%)$ of them were female. OS tumors were located in right/left dis femur, right/left tibia, right foot and shoulder, right/left proximal fibula, right/left humerus, and left pelvic locations. In total, 23 (46\%) patients had less than $90 \%$ and $27(54 \%)$ of them had more than $90 \%$ necrosis. Of the 50 patients, $38(76 \%)$ cases had no metastases and $10(20 \%)$ cases had lung metastases and iliac and lumbar spine metastases was only observed in 1 and 1 (2\%) of cases. Regarding Enneking staging, 38 $(76 \%)$ patients were at stage II and $12(24 \%)$ at stage III.

Correlation between CD133 expression and clinicopatho $\neg$ logical characteristics

CD133 expression was observed in the cytoplasm and membrane of tumor cells and representative areas of immunostained OS tissues are shown in Figure 1. The number of CD133-positive cells considerably varied between different tumor samples. The stained sections were defined as $C D 133$ positive if $C D 133$ expression was detected in more than $10 \%$ of the whole tumor area. As shown in Table 1, CD133 expression was classified based on the mean of $C D 133 \mathrm{H}$-score which was 50 into low and high expression. High expression was found in 13/50 (26\%) of OS cases. Of the 12 patients who showed lung metastasis, 5 cases belong to the high CD133 expression group, and 7 cases were in the low $C D 133$ expression group with marginal trend $(\mathrm{P}=0.06)$. No significant correlation was observed between $C D 133$ expression and

Table 1. Correlation between CD133 Expression and Clinicopathological Factors in the Osteosarcoma Patients

\begin{tabular}{|c|c|c|c|c|c|c|c|}
\hline \multirow[t]{2}{*}{ Patients and tumor characteristics } & \multirow[t]{2}{*}{ No. of cases $(\%)$} & \multicolumn{2}{|c|}{ Expression of CD133 } & \multirow[t]{2}{*}{$P$-value } & \multicolumn{2}{|c|}{ Expression of CXCR4 } & \multirow[t]{2}{*}{$P$-value } \\
\hline & & low & high & & low & high & \\
\hline Mean age (yrs) \pm SD & $19 \pm 8.9$ & & & & & & \\
\hline$\leq 19$ & $24(48)$ & $19(38)$ & $7(14)$ & 1 & $20(40)$ & $6(12)$ & 0.75 \\
\hline$>19$ & $26(52)$ & $18(36)$ & $6(12)$ & & $17(34)$ & $7(14)$ & \\
\hline \multicolumn{8}{|l|}{ Gender } \\
\hline Male & $29(58)$ & $22(44)$ & $7(14)$ & 0.75 & $24(48)$ & $5(10)$ & 0.11 \\
\hline Female & $21(42)$ & $15(30)$ & $6(12)$ & & $13(26)$ & $8(16)$ & \\
\hline \multicolumn{8}{|l|}{ Tumor size } \\
\hline$\leq 8$ & $22(44)$ & $17(34)$ & $5(10)$ & 0.75 & $16(32)$ & $6(12)$ & 1 \\
\hline$>8$ & $28(56)$ & $20(40)$ & $8(16)$ & & $21(42)$ & $7(14)$ & \\
\hline \multicolumn{8}{|l|}{ Enneking stage } \\
\hline II & $17(34)$ & $30(60)$ & $8(16)$ & 0.25 & $29(58)$ & $9(18)$ & 0.7 \\
\hline III & $33(66)$ & $7(14)$ & $5(10)$ & & $8(16)$ & $4(8)$ & \\
\hline \multicolumn{8}{|l|}{ Metastasis } \\
\hline Yes & $12(24)$ & $7(14)$ & $5(10)$ & 0.06 & $8(16)$ & $4(8)$ & 0.7 \\
\hline No & $38(76)$ & $30(60)$ & $8(16)$ & & $29(58)$ & $9(18)$ & \\
\hline \multicolumn{8}{|l|}{ Necrosis $(\%)$} \\
\hline$\leq 90$ & $23(46)$ & $15(30)$ & $8(16)$ & 0.21 & $18(36)$ & $5(10)$ & 0.74 \\
\hline$>90$ & $27(54)$ & $22(44)$ & $5(10)$ & & $19(38)$ & $8(16)$ & \\
\hline
\end{tabular}


Table 2. Correlation between CD133/CXCR4 Phenotypes and Clinicopathological Factors in the Osteosarcoma Patients

\begin{tabular}{|c|c|c|c|c|c|c|}
\hline \multirow[t]{2}{*}{ Patients and tumor characteristics } & \multirow[t]{2}{*}{ No. of cases (\%) } & \multicolumn{4}{|c|}{ CD133/CXCR4 phenotypes } & \multirow[t]{2}{*}{$P$-value } \\
\hline & & $\begin{array}{l}\text { CD133Low/ } \\
\text { CXCR4Low }\end{array}$ & $\begin{array}{l}\text { CD133High/ } \\
\text { CXCR4Low }\end{array}$ & $\begin{array}{l}\text { CD133Low/ } \\
\text { CXCR4High }\end{array}$ & $\begin{array}{l}\text { CD133High/ } \\
\text { CXCR4High }\end{array}$ & \\
\hline Mean age (yrs) $\pm \mathrm{SD}$ & $19 \pm 8.9$ & & & & & \\
\hline$\leq 19$ & $24(48)$ & $16(32)$ & $4(8)$ & $3(6)$ & $3(6)$ & 0.87 \\
\hline$>19$ & $26(52)$ & $15(30)$ & $2(4)$ & $3(6)$ & $4(8)$ & \\
\hline \multicolumn{7}{|l|}{ Gender } \\
\hline Male & $29(58)$ & $20(40)$ & $4(8)$ & $2(4)$ & $3(6)$ & 0.43 \\
\hline Female & $21(42)$ & $11(22)$ & $2(4)$ & $4(8)$ & $4(8)$ & \\
\hline \multicolumn{7}{|l|}{ Tumor size } \\
\hline$\leq 8$ & $22(44)$ & $14(28)$ & $2(4)$ & $3(6)$ & $3(6)$ & 0.94 \\
\hline$>8$ & $28(56)$ & $17(34)$ & $4(8)$ & $3(6)$ & $4(8)$ & \\
\hline \multicolumn{7}{|l|}{ Tumor stage } \\
\hline II & $17(34)$ & $26(52)$ & $3(6)$ & $29(58)$ & $9(18)$ & 0.31 \\
\hline III & $33(66)$ & $5(10)$ & $3(6)$ & $2(4)$ & $2(4)$ & \\
\hline \multicolumn{7}{|l|}{ Lung Metastasis } \\
\hline Yes & $10(20)$ & $3(6)$ & $3(6)$ & $2(4)$ & $2(4)$ & 0.05 \\
\hline No & $40(80)$ & $28(56)$ & $3(6)$ & $4(8)$ & $5(10)$ & \\
\hline \multicolumn{7}{|l|}{ Necrosis $(\%)$} \\
\hline$\leq 90$ & $23(46)$ & $14(28)$ & $4(8)$ & $1(2)$ & $4(8)$ & 0.34 \\
\hline$>90$ & $27(54)$ & $17(34)$ & $2(4)$ & $5(10)$ & $3(6)$ & \\
\hline
\end{tabular}

other clinicopathological characteristics listed in Table 1 and Figure 2.

Correlation between CXCR4 expression and clinicopathological characteristics

The different expression levels of the CXCR4 was observed and indicated in Figure 1. Positive CXCR4 expression was mainly detected in the cell membrane and cytoplasm of OS cells. A small number of OS cases showed CXCR4 expression (36\%) compared to negative group (64\%). The expression of CXCR4 was grouped based on the mean of $C X C R 4 \mathrm{H}$-score as a cut off (mean=31). The expression of CXCR4 is presented in both groups as high (mean $\leq 31)$ and low (mean $>31$ ) expression. As indicated in Table 1, overall CXCR4 expression was not significantly associated with gender,

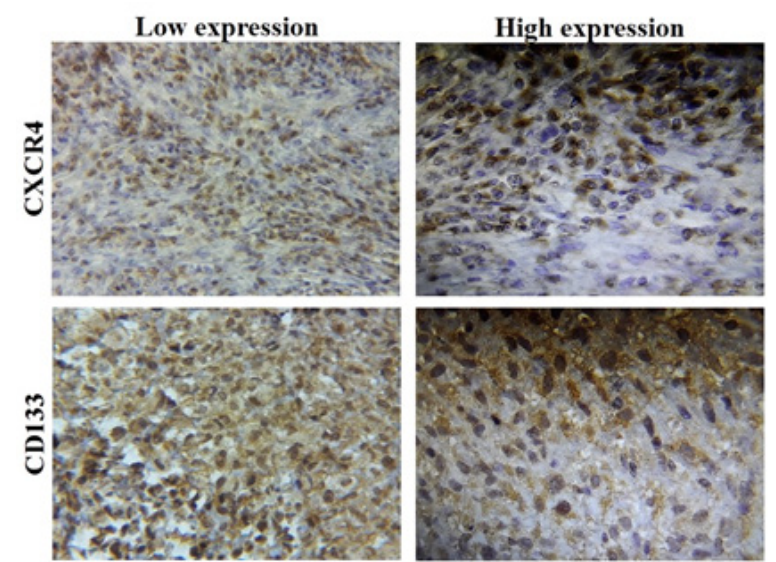

Figure 1. Low and High CD133 and CXCR4 Expression Patterns in Human OS Tissue (Magnification, $400 \times$ ) age, tumor size, necrosis, stage and metastasis $(\mathrm{P}>0.05)$.

Association between CD133/CXCR4 markers expression with clinicopathological features of OS patients

As indicated in Table 2, overall CD133 and CXCR4 examination using Pearson's chi-square showed a significant reciprocal correlation between the expression patterns of the CD133 and CXCR4 $(\mathrm{P}=0.004) . C D 133$ / $C X C R 4$ markers expression were classified into 4 groups including CD113High/CXCR4 Low, CD113 Low/CXCR4 High, low expression of both markers (CD133Low/ $C X C R 4 \mathrm{Low})$, and high expression group (CD133High/

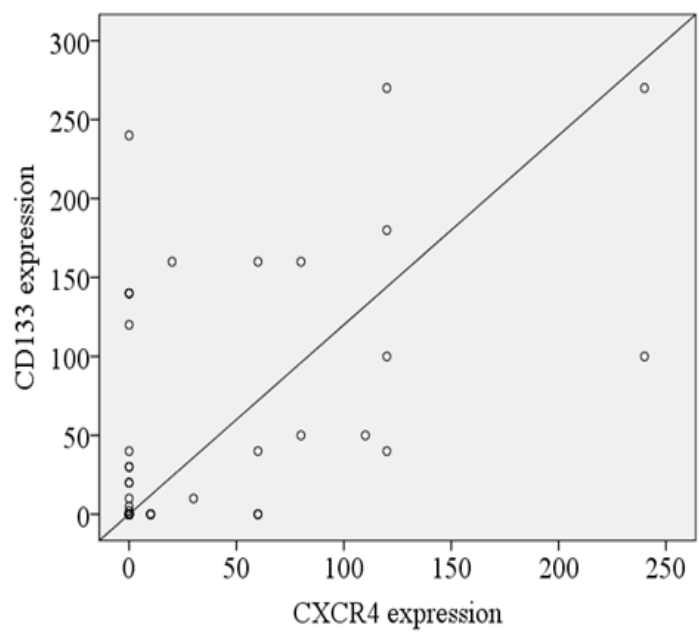

Figure 2. Box-Plot Graphics of CD133 and CXCR4 Expression Scoring (A) in Terms of Tumor Size, Necrosis, Stage and Metastasis. A plot shows mean (horizontal line), the lower and upper lines of the box 75 th and 25 th and outliers (circle) 
CXCR4High). The association between CD133/CXCR4 phenotypes and clinicopathological factors were evaluated by the one-way ANOVA and Tukey's post hoc analysis (Table 2). A significant association was observed between $C D 133 / C X C R 4$ phenotypes and lung metastasis, indicating that $C D 133 / C X C R 4$ expression was positively and equally related to lung metastasis as analyzed by the Tukey's post hoc test $(\mathrm{P}=0.05)$. However, there was no significant association between the expression of $C X C R 4$ and $C D 133$ and any of the other clinicopathological features listed in Table $2(\mathrm{P}>0.05)$.

\section{Discussion}

Osteosarcoma is the most common type of primary malignant bone tumor usually occurs during childhood or adolescence (Xie et al., 2016). Regardless of the long-term survival (68\%), OS shows poor prognosis with high local aggressiveness and tendency for recurrence and metastasis especially to the lungs (Xie et al., 2016).

Therefore, it is essential to elucidate underlying causes and markers involved in OS progression and metastasis and to find new therapeutic targets and approaches. It has been reported that OS metastasis play an important role in promotion of migratory tumor cells and metastatic niche generation at distant organs. Several factors via multifaceted processes and different signaling pathways involve in cancer metastasis ( $\mathrm{Lu}$ et al., 2015b). Many studies have been focused on Chemokines as the main recruiting factors and on chemokine receptors produced by metastatic niche which lead to tumor cell attraction and homing in distant sites (Lazennec et al., 2010; Balkwill et al., 2004; Kryczek et al., 2007). CXCR4 is an important role in the trafficking of normal and malignant cells to distant organs including the lymph nodes, lungs, liver and bone (Micucci et al., 2015). CXCR4 as a CSC marker involved in tumor progression or poor survival of OS has been suggested by some studies (Sand et al., 2015; Xie et al., 2016). It has been reported that the high expression of CSC marker CXCR4 correlated with significant prognostic value and therapeutic importance of RCC patients (D'Alterio et al., 2010). It has been revealed that CSCs exist in primary OS and cell lines derived from human OS tumors (Tirino et al., 2008; Gibbs et al., 2005). $\mathrm{He}$ at al showed that mRNA of the stemness genes such as octamer-binding transcription factor4 (Oct-4) and NANOG, and the metastasis-related receptor CXCR4 were highly expressed in CD133+ OS cells (He et al., 2012). They also showed that $C D 133$ expression as an independent prognostic factor was associated with lung metastasis and poor prognosis of OS patients (He et al., 2012). The current study was performed to assess the tissue expression pattern of $C D 133$ as a type of CSC marker and CXCR4 as a metastasis-related marker in OS tumors using immunostaining method. To this end, we first defined that if OS tumors could express CD133 and CXCR4. In our patient data, we found that high expression of $C D 133$ was partially associated with lung metastasis as a worse prognostic factor of OS. In line with our findings, He et al reported that high expression of CD133 in OS tissues was associated with the high lung metastasis risk of OS $(\mathrm{He}$ et al., 2012). Based on previously published findings by Zambo et al., (2016) CD133 along with other CSC markers including nestin and $A B C G 2$ could be represented CSC phenotype in bone and soft tissue sarcomas. It has also been shown that high expression of $C X C R 4$ along with other stemness genes can affect the properties of $C D 133+$ cells invasion and migration supporting the proposed link between CD133 expression and CSC characteristics (He et al., 2012). In a study performed by Lu et al the higher CXCR 4 and $\beta$ catenin.

mRNA and protein expression rate was observed in OS cases than in the osteochondroma cases which were significantly correlated with the clinical Enneking stage, metastasis and survival of OS (Lu et al., 2015b). In other study of Lu et al significantly higher expression of CXCR4 and $\beta$-catenin mRNA and protein was found in OS tissues compared to the adjacent non-neoplastic areas ( $\mathrm{Lu}$ et al., 2015c). Another animal study showed that higher percentage of CXCR4-expressing lung metastatic cells compared to primary tumors is due to the important role of CXCL12/CXCR4 axis in late metastasis (Neklyudova et al., 2016). Guo et al was revealed that coexpression of CXCR4 with other factors such as HIF-1 $\alpha$ through the hypoxia-HIF-1 $\alpha$-CXCR4 pathway has crucial function during the migration of human OS cells representing a novel targeting strategy specially for OS patients with distant metastasis (Guo et al., 2014). In Bai et al study using 22 patients with chondrosarcoma, higher CXCR4 expression levels was observed in high-grade cells than in low-grade specimens (Bai et al., 2011). They suggested that their findings needed to be expanded and to be confirmed by a larger number of cases (Bai et al., 2011). High expression of CXCR4 was revealed in canine OS cells with its signaling role in cell migration (Fan et al., 2008). To date, many studies have focused on the detection and possible prognostic significance of $C X C R 4$ as a reliable CSC marker in other cancers (Retz et al., 2005; Zhang et al., 2012). However, relatively little is known about the prognostic significance of $C X C R 4$ individually or in combination with other CSC markers in human OS. As previously revealed data were achieved using animal model, sarcoma cell lines and molecular evaluation (Walter et al., 2014; Zhang et al., 2013; de Nigris et al., 2008; Fan et al., 2008), our study has been more focused on the protein expression levels of two CSC markers CD133 and CXCR4 using immunohistochemical detection in the human OS tissues. Furthermore, we examined the possible relationship of $C D 133$ and $C X C R 4$ expression levels with metastasis as the main patients' characteristic. Our immunohistochemical analysis of Iranian OS patients, for the first time, showed that despite the expression of CXCR4 in tissues, no significant relationship between its expression and other clinical factors was observed. This discrepancy may be the result of variations in clones of antibodies, cut-off point and population study.

Co-expression of nestin, $C D 133$ and $A B C G 2$ as putative CSC markers has been studied in different type of sarcomas (Zambo et al., 2016). Co-expression of both CSCs markers of CD133 and CXCR4 has been presented in a variety of cancers, including colon cancer as markers for metastatic potential and poor prognosis 
(Silinsky et al., 2013; Li et al., 2015; Margolin et al., 2015), esophageal squamous cell carcinoma as a novel marker for predicting the poor prognosis of patients $(\mathrm{Lu}$ et al., 2015a), non-small cell lung cancer (NSCLC) as a prognostic marker via CD133/CXCR4/EMT axis (Tu et al., 2017), pancreatic adenocarcinoma (PA) (Mizukami et al., 2014), anaplastic thyroid carcinoma (ATC) (Yun et al., 2014) and renal cancer (RCC) (D’Alterio et al., 2010). Our study represented the first combined analysis of two putative CSCs markers expression together in human OS tissues and displayed their promising predictive values in OS. We found that CD34High/CD117 High phenotype was more frequent in OS cases.

Among 50 patient tissues, concomitant high CD133-CXCR4 expression accounts for 20.78\% (32/154). We also observed a positive significant correlation between CD133/CXCR4 phenotype with OS lung metastasis. No significant correlation was found between co-expression of $C D 133 / C X C R 4$ with other clinicopathologic variables. The concomitant high CD133-CXCR4 expression might be a novel marker for predicting the poor prognosis of patients with OS, and CD133 and CXCR4 may serve as potential therapeutic targets. Our preliminary data would argue for concomitant CD133/CXCR4 immunostaining as a potential marker for biologic aggressiveness in estosarcoma of bone.

In conclusion, based on the here reported results regarding the synergistic effect of CD133 and CXCR4 on metastasis and progression of OS, these two markers might be considered as potential therapeutic targets and favored over anti-metastatic systemic therapy.

\section{Acknowledgments}

This study was supported by Iran University of Medical Sciences. We thank shafa yahyaian hospital for their cooperation.

\section{Ethical approval}

This article does not contain any studies with human participants performed by any of the authors.

\section{Conflict of interest}

All authors declare no conflict of interest.

\section{References}

Adhikari A, Agarwal N, Wood B, et al (2010). CD117 and Stro-1 identify osteosarcoma tumor-initiating cells associated with metastasis and drug resistance. Cancer Res, 70, 4602-12.

Bai S, Wang D, Klein M, Siegal G (2011). Characterization of CXCR4 expression in chondrosarcoma of bone. Archives of pathology \& laboratory medicine, 135, 753-758.

Balkwill F (2004). Cancer and the chemokine network. Nat Rev Cancer, 4, 540.

Brown K, Tellez-gabriel M, Heymann D (2017). Cancer stem cells in osteosarcoma. Cancer Lett, 386, 189-95.

D'alterio C, Cindolo L, Portella L, et al (2010). Differential role of CD133 and CXCR4 in renal cell carcinoma. Cell Cycle, 9, 4492-4500.

De nigris F, Rossiello R, Schiano C, et al (2008). Deletion of Yin Yang 1 protein in osteosarcoma cells on cell invasion and CXCR4/angiogenesis and metastasis. Cancer Res, 68 , 1797-1808.

Diestra J, Scheffer G, Catala I, et al (2002). Frequent expression of the multi-drug resistance-associated protein BCRP/MXR/ $\mathrm{ABCP} / \mathrm{ABCG} 2$ in human tumours detected by the BXP-21 monoclonal antibody in paraffin-embedded material. $J$ Pathol, 198, 213-9.

Fan T, Barger A, Fredrickson R, et al (2008). Investigating CXCR4 expression in canine appendicular osteosarcoma. $J$ Vet Intern Med, 22, 602-8.

Foroozan M, Roudi R, Abolhasani M, et al (2017). Clinical significance of endothelial cell marker CD34 and mast cell marker CD117 in prostate adenocarcinoma. Pathol Res Pract, 213, 612-8.

Gelmini S, Mangoni M, Serio M, et al (2008). The critical role of SDF-1/CXCR4 axis in cancer and cancer stem cells metastasis. $J$ Endocrinol Invest, 31, 809-19.

Gibbs C, Kukekov V, Reith J, et al (2005). Stem-like cells in bone sarcomas: implications for tumorigenesis. Neoplasia, 7, 967-76.

Guo M, Cai C, Zhao G, et al (2014). Hypoxia promotes migration and induces CXCR4 expression via HIF-1 $\alpha$ activation in human osteosarcoma. PLoS One, 9, e90518.

He A, Qi W, Huang Y, et al (2012). CD133 expression predicts lung metastasis and poor prognosis in osteosarcoma patients: A clinical and experimental study. Exp Ther Med, 4, 435-41.

Hermann P, Huber S, Herrler T, et al (2007). Distinct populations of cancer stem cells determine tumor growth and metastatic activity in human pancreatic cancer. Cell Stem Cell, 1, 313-23.

Honoki K, Fujii H, Kubo A, et al (2010). Possible involvement of stem-like populations with elevated ALDH1 in sarcomas for chemotherapeutic drug resistance. Oncol Rep, 24, 501-5.

Jung Y, Wang J, Schneider A, et al (2006). Regulation of SDF-1 (CXCL12) production by osteoblasts; a possible mechanism for stem cell homing. Bone, 38, 497-508.

Kazama S, Kishikawa J, Kiyomatsu T, et al (2018). Expression of the stem cell marker CD133 is related to tumor development in colorectal carcinogenesis. Asian J Surg, 41, 274-8.

Keymoosi H, Gheytanchi E, Asgari M, et al (2014). ALDH1 in combination with CD44 as putative cancer stem cell markers are correlated with poor prognosis in urothelial carcinoma of the urinary bladder. Asian Pac J Cancer Prev, 15, 2013-20.

Kryczek I, Wei S, Keller E, et al (2007). Stroma-derived factor (SDF-1/CXCL12) and human tumor pathogenesis. Am J Physiol Cell Physiol, 292, 987-95.

Kucia M, Reca R, Miekus K, et al (2005). Trafficking of normal stem cells and metastasis of cancer stem cells involve similar mechanisms: Pivotal Role of the SDF-1-CXCR4 Axis. Stem Cells, 23, 879-94.

Lazennec G, Richmond A (2010). Chemokines and chemokine receptors: new insights into cancer-related inflammation. Trends Mol Med, 16, 133-44.

Li X, Guo X, Yang Y, Liu A (2015). Effect of CXCR4 and CD133 co-expression on the prognosis of patients with stage II III colon cancer. Asian Pac J Cancer Prev, 16, 1073-6.

Liu G, Yuan X, Zeng Z, et al (2006). Analysis of gene expression and chemoresistance of CD133+ cancer stem cells in glioblastoma. Mol Cancer, 5, 67.

Lu C, Xu F, Gu J, et al (2015a). Clinical and biological significance of stem-like CD133+ CXCR4+ cells in esophageal squamous cell carcinoma. J Thorac Cardiovasc Surg, 150, 386-95.

Lu Y, Guan G, Chen J, et al (2015b). Aberrant CXCR4 and $\beta$-catenin expression in osteosarcoma correlates with patient survival. Oncol Lett, 10, 2123-9.

Lu Y, Hu B, Guan G, et al (2015c). SDF-1/CXCR4 promotes 
F5M2 osteosarcoma cell migration by activating the Wnt/ $\beta$ catenin signaling pathway. Med Oncol, 32, 194.

Madjd Z, Erfani E, Gheytanchi E, et al (2016). Expression of CD133 cancer stem cell marker in melanoma: A systematic review and meta-analysis. Int J Biol Markers, 31, 118-25.

Madjd Z, Gheytanchi E, Erfani E, Asadi-lari M (2013). Application of stem cells in targeted therapy of breast cancer: a systematic review. Asian Pac J Cancer Prev, 14, 2789-800.

Margolin D, Myers T, Zhang X, et al (2015). The critical roles of tumor-initiating cells and the lymph node stromal microenvironment in human colorectal cancer extranodal metastasis using a unique humanized orthotopic mouse model. FASEB J, 29, 3571-81.

Micucci C, Matacchione G, Valli D, Orciari S, Catalano A(2015). HIF2 $\alpha$ is involved in the expansion of CXCR4-positive cancer stem-like cells in renal cell carcinoma. Br J Cancer, 113, 1178.

Mizukami T, Kamachi H, Mitsuhashi T, et al (2014). Immunohistochemical analysis of cancer stem cell markers in pancreatic adenocarcinoma patients after neoadjuvant chemoradiotherapy. BMC Cancer, 14, 687.

Müller A, Homey B, Soto H, et al (2001). Involvement of chemokine receptors in breast cancer metastasis. Nature, 410, 50 .

Murase M, Kano M, Tsukahara T, et al (2009). Side population cells have the characteristics of cancer stem-like cells/cancerinitiating cells in bone sarcomas. Br J Cancer, 101, 1425.

Neklyudova O, Arlt M, Brennecke P, et al (2016). Altered CXCL12 expression reveals a dual role of CXCR4 in osteosarcoma primary tumor growth and metastasis. J Cancer Res Clin Oncol, 142, 1739-50.

Ottaviani G, Jaffe N (2009). The epidemiology of osteosarcoma. Pediatric and adolescent osteosarcoma. Springer, 152, 3-13.

Perissinotto E, Cavalloni G, Leone F, et al (2005). Involvement of chemokine receptor 4/stromal cell-derived factor 1 system during osteosarcoma tumor progression. Clin Cancer Res, 11, 490-7.

Retz M, Sidhu S, Blaveri E, et al (2005). CXCR4 expression reflects tumor progression and regulates motility of bladder cancer cells. Int J Cancer, 114, 182-9.

Roudi R, Korourian A, Shariftabrizi A, Madjd Z (2015). Differential expression of cancer stem cell markers ALDH1 and CD133 in various lung cancer subtypes. Cancer Invest, 33, 294-302.

Sana J, Zambo I, Skoda, et al (2011). CD133 expression and identification of CD133/nestin positive cells in rhabdomyosarcomas and rhabdomyosarcoma cell lines. Anal Cell Pathol, 34, 303-18.

Sand L, Scotlandi K, Berghuis D, et al (2015). CXCL14, CXCR7 expression and CXCR4 splice variant ratio associate with survival and metastases in Ewing sarcoma patients. Eur $J$ Cancer, 51, 2624-33.

Saur D, Seidler B, Schneider G, et al (2005). CXCR4 expression increases liver and lung metastasis in a mouse model of pancreatic cancer. Gastroenterology, 129, 1237-50.

Sedaghat S, Gheytanchi E, Asgari M, et al (2017). Expression of cancer stem cell markers OCT4 and CD133 in transitional cell carcinomas. Appl Immunohisto M M, 25, 196-202.

Silinsky J, Grimes C, Driscoll T, et al (2013). CD 133+ and CXCR4+ colon cancer cells as a marker for lymph node metastasis. J Surg Res, 185, 113-8.

Song T, Dou C, Jia Y, Tu K, Zheng X (2015). TIMP-1 activated carcinoma-associated fibroblasts inhibit tumor apoptosis by activating SDF1/CXCR4 signaling in hepatocellular carcinoma. Oncotarget, 6, 12061.

Sun X, Cheng G, Hao M, et al (2010). CXCL12/CXCR4/CXCR7 chemokine axis and cancer progression. Cancer Metastasis
Rev, 29, 709-22.

Suvà M, Riggi N, Stehle J, et al (2009). Identification of cancer stem cells in Ewing's sarcoma. Cancer Res, 69, 1776-81.

Terry J, Nielsen T(2010). Expression of CD133 in synovial sarcoma. Appl Immunohisto M M, 18, 159-65.

Tirino V, Desiderio V, D'aquino R, et al (2008). Detection and characterization of CD133+ cancer stem cells in human solid tumours. PLoS One, 3, e3469.

Tirino V, Desiderio V, Paino F, et al (2011). Human primary bone sarcomas contain $\mathrm{CD} 133+$ cancer stem cells displaying high tumorigenicity in vivo. FASEB J, 25, 2022-30.

Tobe R, Martin G, Moriichi A, Wu B, Mori R (2017). Cost-effectiveness analysis of neonatal screening of critical congenital heart defects in China. Medicine, 96, e868.

Tu Z, Xie S, Xiong M, et al (2017). CXCR4 is involved in CD133-induced EMT in non-small cell lung cancer. Int $J$ Oncol, 50, 505-14.

Walter I, Wolfesberger B, Miller I, et al (2014). Human osteosarcoma cells respond to sorafenib chemotherapy by downregulation of the tumor progression factors S100A4, CXCR4 and the oncogene FOS. Oncol Rep, 31, 1147-56.

Wang L, Jin J, Lv F, et al (2015). Norepinephrine attenuates CXCR4 expression and the corresponding invasion of MDA-MB-231 breast cancer cells via $\beta 2$-adrenergic receptors. Eur Rev Med Pharmacol Sci, 19, 1170-81.

Wang L, Park P, Zhang H, La M, Lin C (2011). Prospective identification of tumorigenic osteosarcoma cancer stem cells in OS99-1 cells based on high aldehyde dehydrogenase activity. Int J Cancer, 128, 294-303.

Xie T, Ren H, Lin H, et al (2016). Sinomenine prevents metastasis of human osteosarcoma cells via $\mathrm{S}$ phase arrest and suppression of tumor-related neovascularization and osteolysis through the CXCR4-STAT3 pathway. Int J Oncol, 48, 2098-2112.

Yan G, Lv Y, Guo Q (2016). Advances in osteosarcoma stem cell research and opportunities for novel therapeutic targets. Cancer Lett, 370, 268-274.

Yin S, Li J, Hu C, et al (2007). CD133 positive hepatocellular carcinoma cells possess high capacity for tumorigenicity. International journal of cancer, 120, 1444-1450.

Yun J, Kim Y, Choe J, et al (2014). Expression of cancer stem cell markers is more frequent in anaplastic thyroid carcinoma compared to papillary thyroid carcinoma and is related to adverse clinical outcome. J Clin Pathol, 67, 125-33.

Zambo I, Hermanova M, Zapletalova D, et al (2016). Expression of nestin, CD133 and ABCG2 in relation to the clinical outcome in pediatric sarcomas. Cancer Biomarkers, 17, $107-16$.

Zeelenberg I, Ruuls-van stalle L, Roos E (2003). The chemokine receptor CXCR4 is required for outgrowth of colon carcinoma micrometastases. Cancer Res, 63, 3833-9.

Zhang P, Dong L, Yan K, et al (2013). CXCR4-mediated osteosarcoma growth and pulmonary metastasis is promoted by mesenchymal stem cells through VEGF. Oncol Rep, $\mathbf{3 0}$, 1753-61.

Zhang S, Han Z, Jing Y, et al (2012). CD133+ CXCR4+ colon cancer cells exhibit metastatic potential and predict poor prognosis of patients. BMC Med, 10, 85 .

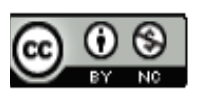

This work is licensed under a Creative Commons AttributionNon Commercial 4.0 International License. 